\title{
Histopathological analysis of hysterectomy specimens: one year study
}

\author{
Baral R ${ }^{1}$, Sherpa $\mathrm{P}^{1}$, Gautam $\mathrm{D}^{1}$ \\ ${ }^{I}$ Department of Pathology, Patan Academy of Health Sciences, Lalitpur, Nepal.
}

\section{Keywords: \\ Adenocarcinoma; Endometrioid; Hysterectomy; Leiomyoma}

\begin{abstract}
Background: The uterus is prone to develop several non-neoplastic and neoplastic conditions during the life time of a woman. The aim of this study is to study the histopathological features of varied uterine lesions, their profile and distribution of different lesions in relation of age.

Materials and Methods: This is a histopathological database analysis of hysterectomy specimen of one year 2011/12 in Patan Hospital. The variables studied were age and histopathological diagnosis. SPSS version 16 was used as an analytical tool.

Results: A total of 3576 histopathology samples were received in this period. There were 1173 gynaecology samples during this period out of which $22 \%$ (261 cases) were that of hysterectomy. Histopathology diagnosis showed Leiomyoma in 48.6\% (127 cases), Adenomyosis was seen in 10.3\% (27 cases), Endometrioid Adenocarcinoma was seen in 1.14\% (3 cases).
\end{abstract}

Conclusion: A large number of hysterectomy specimens had no significant findings. However, adenomyosis, leiomyomya and adenocarcinoma are also found which may be the cause of abnormal uterine bleeding.

\section{INTRODUCTION}

The uterus is prone to develop several non-neoplastic and neoplastic conditions during the life time of a woman. The uterus consists of endometrium and myometrium which is under the influence of different hormones periodically. Many studies have shown many different types of lesions in the ovaries and fallopian tubes. The cervix is prone to many

\section{Correspondence:}

Dr. Reetu Baral, MBBS, MD

Associate Professor, Department of Pathology

Patan Academy of Health Sciences, Lalitpur, Nepal.

E-mail: reetubaral@pahs.edu.np non-neoplastic and neoplastic conditions which are mostly seen in the reproductive age group. All these diseases are seen across all age groups and contribute significantly to increased morbidity and mortality amongst women. ${ }^{1}$

Most common complaints presented are per vaginal bleeding, vaginal discharge, pain abdomen, irregular menstruation, postmenopausal bleeding, mass per abdomen, something coming out of vagina etc. ${ }^{2}$ Many treatment options are available including medical and conservative surgical treatments but hysterectomy remains one of the most preferred method to manage gynecological disorders. 
This study is done to study various gross and histopathological findings in uterus and cervix of the hysterectomy specimens received in the histopathology laboratory. The objective of the study was to study the histopathological features of varied uterine lesions, their profile and distribution of different lesions in relation of age.

\section{MATERIALS AND METHODS}

This is a retrospective cross sectional study done in the Department of Pathology in Patan Academy of Health Sciences, Patan Hospital, Lalitpur, Nepal. The study was conducted in the histopathology samples received in the year 2011/2012. A total of 3576 histopathology samples were received in this period. There were 1173 gynaecology samples during this period out of which $22 \%$ (261 cases) were that of hysterectomy. The specimens were grossed by the pathologists; the tissues underwent processing in the tissue processor overnight. Blocks were made and sections were cut into 4 micron thickness in the microtome. These sections were stained by the Histotechnicians by routine stain of hematoxylin and eosin. Each slide was then meticulously examined by Pathologists. Inclusion criteria were that all hysterectomy samples received in the pathology lab were included in the study. Exclusion criteria were specimens with incomplete requisition forms and subtotal hysterectomy. All the findings were recorded in the database. Graphical method of normality test is done to compare data distribution by age. Test requisition form without age of the patients was excluded. Analyzable data from histopathology database were analysed using SPSS 16.0 version.

\section{RESULTS}

Endometrium was the most biopsied specimen with $43 \%$ (503 cases). Total 266 hysterectomy specimens were received in the lab in 2011-2012. Out of these 5 cases had to be excluded from the study due to lack of complete information in the requisition form. Maximum number of women who underwent the gynecological biopsy was of age group 21-30 years comprising of 30\% (346 cases). In $4.3 \%$ (51 cases) age was not mentioned.

Histopathology diagnosis showed Leiomyoma in 48.6\% (127 cases), Adenomyosis was seen in 10.3\% (27 cases), Endometrioid Adenocarcinoma was seen in $1.14 \%$ (3 cases). In $10.7 \%$ (6 cases) of cervix chronic cervicitis with Koilocytotic changes were seen. The most common pathology seen in the ovary was endometriosis $(3.8 \%, 10$ cases) followed by Mature Cystic Teratoma in 3.5\% ( 9 cases), Serous Cystadenoma in 3.5\% (9 cases) and Mucinous Cystadenoma in 2\% (5 cases). Neoplastic conditions like Serous Cystadenocarcinoma (1.2\%, 3 cases), Krukenberg tumor and Immature teratoma (1\%, 2 cases) were also seen in lesser percentage of patients.

\section{DISCUSSION}

Hysterectomy is one of the common surgeries performed in the gynecology department. It is done for various causes which includes lifesaving methods in case of rupture uterus during delivery to patient's satisfaction who has been suffering from abnormal uterine bleeding. Hysterectomy is also a procedure which gives permanent relief in many nonneoplastic conditions.

This study was conducted to analyze the patterns of lesions in hysterectomy specimens in our institution, correlate the findings with the clinical indications and to compare our findings with those of other workers.

In the present study maximum number of patients i.e is $41.3 \%$ were seen in the age group of $41-50$ years. In an analysis of 150 cases by Harshal A. et $a^{3}$ most cases were if age group of 41- 50 years. Various studies done by Rather GR et $\mathrm{al}^{4}$, Ramchandran $\mathrm{T}$ et $\mathrm{al}^{5}$ and Ajmera et $\mathrm{al}^{6}$ had similar findings. Most common surgical procedure for hysterectomy was Abdominal Hysterectomy $(79 \%, \mathrm{n}=205)$ and Vaginal hysterectomy $(21 \%, \mathrm{n}=56)$. All the vaginal hysterectomy was done for second and third degree Uterovaginal prolapse. In a study done by Domblae $\mathrm{V}$ in India had shown $69.6 \%$ cases of abdominal hysterectomy and $30.4 \%$ of vaginal hysterectomy. ${ }^{2}$

Atrophic endometrium was seen in 20 cases, all except one was diagnosed in vaginal hysterectomy done in the postmenopausal women. Endometrial polyp was seen in 5 cases. Endometrial adenocarcinoma was seen in 1.1\% $(\mathrm{n}=3)$ in the hysterectomy specimens. Two cases were of age group $40-50$ and one was 60-70 years. All the cases had presented with postmenopausal bleeding. This finding is similar to other studies. ${ }^{2}$

Leiomyoma is the most common lesion myometrial lesion in this study with $48.6 \%$ cases. Most of the studies showed leiomyoma as the most common presentation. ${ }^{1,2}$ Most of the cases of leiomyoma present with PV bleeding, dysmenorrhea, mass in the lower abdominal area etc. Adenomyosis is a diagnosis made after hysterectomy by histopathology. Since the symptoms of adenomyosis are vague it is undiagnosed unless hysterectomy specimens undergo histopathology tests. In a study done by Rhizvi $\mathrm{G}$ et.al perimenopausal age (41-50 years) group, adenomyosis was the commonest pathology $46.34 \%$ followed by leiomyoma $41.46 \%$ which is similar to this study. ${ }^{7}$ In our study Adenomyosis with leiomyoma is seen in $4.6 \%$ ( 12 cases). This is similar to study done by DhomblaeV ${ }^{2}$. However, other studies have shown a larger percentage of patients presenting with dual pathology. ${ }^{3}$ Chronic cervicitis is the most common finding in the cervix histopathologically. In our study $29 \%$ of the cases were chronic cervicitis. This finding is similar to other studies. ${ }^{8}$ In $10.7 \%$ cases koilocytotic changes were seen. 
This is the microscopic changes seen in HPV infection and is of considerable importance in view of association of carcinoma cervix and HPV. Squamous cell carcinoma is seen only in $1 \%$ of the cases. This maybe because squamous cell carcinoma diagnosed in the cervical biopsy are referred to centers with oncology services.

Maximum number of cases seen in ovary was of mature cystic teratoma with $3.5 \%$ (9 cases). Only two malignant conditions were seen in the ovary like Krukenberg tumor and Serous cystadenocarcinoma. In the fallopian tube $0.8 \%$ ( 2 cases) were chronic nonspecific salpingitis and 2 paratubal cysts. Inflammatory lesions were seen most in other studies too. ${ }^{9}$ Neoplastic conditions were not seen in fallopian tubes. In the study done on histopathological lesions of fallopian tubes $69 \%$ cases were within normal limit similar to our study. ${ }^{10}$

\section{CONCLUSION}

Hysterectomy is a surgical procedure which is commonly practiced. The question arises that whether the histopathologically detected benign lesions do need hysterectomy or not. Focus of malignancies is sometimes detected in the clinically negative specimens. In this study a large number of hysterectomy specimens had no significant findings.

\section{REFERENCES}

1. Forae GD, Aligbe JU. Histopathological patterns of endometrial lesions in patients with abnormal uterine bleeding in a cosmopolitan population. J Basic Clin Reprod Sci 2013;2:101-4. Crossref

2. Domblae V, Gundalli S. International Journal of Science and Research (IJSR) ISSN (Online): 2319-7064.

3. Patil HA., Suresh AP, Mahajan V. MVP Journal of Medical Sciences. 2015;2:26-29. Crossref

4. Rather GR, Gupta Y, Bharadwaj S. Patterns of lesions in hysterectomy specimens; a prospective study. J K Science. 2013;15:63-8.

5. Ramachandran TS, Sinha R, Subramanium. Correlation between clinic pathological and ultrasonographic findings. Journal of Clinical Diagnosis and Research. 2011;5:734-40.

6. Ajmera SK, Mettler L, Jonat W. Operative spectrum of hysterectomy in a German university hospital. J Obstet Gynecol India. 2006;56:59-63.

7. Rizvi G, Pandey H, Pant H, Chufal SS, Pant P. Histopathological correlation of adenomyosis and leiomyoma in hysterectomy specimens as the cause of abnormal uterine bleeding in women in different age groups in the Kumaon region: A retroprospective study. J Mid-life Health 2013;4:2730. Crossref

8. Saravanan S, Arnold J, Arul P. Histomorphological Spectrum of Lesions of the Cervix, A Retrospective Study in a Tertiary Care Hospital. Journal of Evolution of Medical and Dental Sciences 2015;4:10326-9. Crossref

9. Bhagwan IN, Harke AB, Malpani MR, Deshmukh SD. Histopathological study of Spectrum of lesions encountered in the Fallopian Tube. J Obstet Gynecol Ind 2004;54:379-82.

10. Gon S, Basu A, Majumdar B, Das TK, Sengupta M, Ghosh D. Spectrum of histopathological lesions in the fallopian tubes. Journal of Pathology of Nepal 2013;3:356-60. Crossref 